\title{
Electrochemical Generation of Cationic Pd Catalysts and Pd/TEMPO Double-Mediatory Electrooxidative Wacker-type Reactions
}

Koichi Mitsudo, ${ }^{*}$ Takashi Kaide, Eriko Nakamoto, Kenta Yoshida, and Hideo Tanaka*

Division of Chemistry and Biochemistry, Graduate School of Natural Science and Technology, Okayama University, 3-1-1 Tsushima-Naka, Okayama 700-8530, Japan

\section{General}

${ }^{1} \mathrm{H}$ and ${ }^{13} \mathrm{C}$ NMR spectra were recorded on Varian GEMINI $200\left({ }^{1} \mathrm{H} 200 \mathrm{MHz},{ }^{13} \mathrm{C} 50 \mathrm{MHz}\right)$ spectrometer in $\mathrm{CD}_{3} \mathrm{NO}_{2}$ or $\mathrm{CDCl}_{3}$ using TMS or residual solvent as internal standard. IR spectra were recorded on a JASCO FT/IR-4100 spectrophotometer. Unless otherwise noted, all reactions were performed under air. Unless otherwise noted, all materials were obtained from commercial suppliers and used without further purification. Dried acetonitrile $\left(\mathrm{CH}_{3} \mathrm{CN}\right)$ over $\mathrm{CaH}_{2}$ was used in the synthesis of palladium complexes. Unless otherwise noted, all reactions were performed under air.

\section{General Procedure for Electrochemical Preparation of Cationic Palladium Complex}

The anodic oxidation was carried out in an H-type divided cell (glass filter) equipped with two platinum electrodes $\left(1.0 \times 1.5 \mathrm{~cm}^{2}\right)$. In the anodic chamber was placed a solution of $\mathrm{Pd}(\mathrm{OAc})_{2}(22 \mathrm{mg}$, $0.1 \mathrm{mmol})$ in $0.2 \mathrm{M} \mathrm{Bu}_{4} \mathrm{NBF}_{4} / \mathrm{CH}_{3} \mathrm{CN}(5.0 \mathrm{~mL})$. In the cathodic chamber was placed $0.2 \mathrm{M}$ $\mathrm{Bu}_{4} \mathrm{NBF}_{4} / \mathrm{CH}_{3} \mathrm{CN}(5.0 \mathrm{~mL})$. Under argon, the constant current electrolysis $(10 \mathrm{~mA})$ was carried out at room temperature with magnetic stirring until $3.0 \mathrm{~F} / \mathrm{mol}$ of electricity was passed. After the electrolysis, the anodic solution was poured into dry $\mathrm{Et}_{2} \mathrm{O}(10 \mathrm{~mL})$. The resulting pale yellow precipitate was washed with dry $\mathrm{Et}_{2} \mathrm{O}$ and dried under reduced pressure to afford $\left[\mathrm{Pd}\left(\mathrm{CH}_{3} \mathrm{CN}\right)_{4}\right]\left[\mathrm{BF}_{4}\right]_{2} \mathbf{1 a}(26 \mathbf{m g}, 58 \%)$.

$\left[\mathbf{P d}\left(\mathbf{C H}_{3} \mathbf{C N}\right)_{4}\right]\left[\mathbf{B F}_{4}\right]_{2}(\mathbf{1 a}):{ }^{1}{ }^{1} \mathrm{H}$ NMR $\left(200 \mathrm{MHz}, \mathrm{CD}_{3} \mathrm{NO}_{2}\right) \delta 2.66$ (s, 12H); IR (KBr) 2359, 2342, $1084 \mathrm{~cm}^{-1}$. Anal. Calcd for $\mathrm{C}_{8} \mathrm{H}_{12} \mathrm{~B}_{2} \mathrm{~F}_{8} \mathrm{~N}_{4} \mathrm{Pd}: \mathrm{C}, 21.63 ; \mathrm{H}, 2.72 ; \mathrm{N}, 12.61$. Found: $\mathrm{C}, 21.01 ; \mathrm{H}, 2.76 ; \mathrm{N}$, 12.02 .

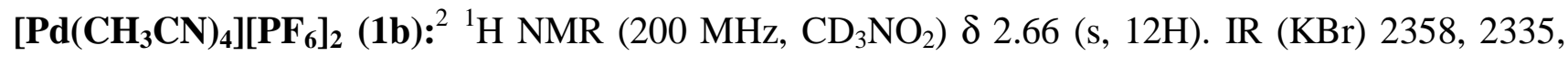
$2359 \mathrm{~cm}^{-1}$. Anal. Calcd for $\mathrm{C}_{8} \mathrm{H}_{12} \mathrm{~F}_{12} \mathrm{~N}_{4} \mathrm{P}_{2} \mathrm{Pd}$ : C, 17.14; H, 2.16; N, 9.99. Found: C, 18.09; H, 2.64; N, 9.94.

$\left[\mathbf{P d}\left(\mathrm{CH}_{3} \mathbf{C N}\right)_{4}\right]\left[\mathrm{ClO}_{4}\right]_{2}(\mathbf{1 c}):{ }^{1} \mathrm{H} \mathrm{NMR}\left(200 \mathrm{MHz}, \mathrm{CD}_{3} \mathrm{NO}_{2}\right) \delta 2.67$ (s, 12H); IR (KBr) 2361, 2334, $1143,1109,1089 \mathrm{~cm}^{-1}$.

(1) Yuan, J.-C.; Lu, S.-J. Organometallics 2001, 20, 2697-2703.

(2) Sommer, R. D.; Rheingold, A. L.; Goshe, A. J.; Bosnich, B.; J. Am. Chem. Soc. 2001, 123, 3940-3952. 


\section{Typical Procedure for Pd/TEMPO Double-Mediatory Electrooxidative Wacker-type Reaction}

The anodic oxidation was carried out in an H-type divided cell equipped with two platinum electrodes $\left(1.0 \times 1.5 \mathrm{~cm}^{2}\right)$. In the anodic chamber were placed 1-dodecene $\mathbf{2 a}(85 \mathrm{mg}, 0.5 \mathrm{mmol}), \mathrm{Pd}(\mathrm{OAc})_{2}(12 \mathrm{mg}$, $0.05 \mathrm{mmol}$ ), and TEMPO (22 $\mathrm{mg}, 0.14 \mathrm{mmol})$ in $0.05 \mathrm{M} \mathrm{NaClO}_{4}$ solution of $\mathrm{CH}_{3} \mathrm{CN}_{2} \mathrm{H}_{2} \mathrm{O}(8.75 / 1.25$ $\mathrm{mL})$. In the cathodic chamber was placed $0.05 \mathrm{M} \mathrm{NaClO}_{4}$ solution of $\mathrm{CH}_{3} \mathrm{CN} / \mathrm{H}_{2} \mathrm{O}(8.75 / 1.25 \mathrm{~mL})$. The constant current electrolysis ( $5 \mathrm{~mA}$ ) was carried out at room temperature with magnetic stirring until 3.0 $\mathrm{F} / \mathrm{mol}$ of electricity was passed. To the resulting mixture was added aq sat. $\mathrm{NaCl}(5 \mathrm{~mL})$ and extracted with EtOAc $(3 \times 5 \mathrm{~mL})$. The combined organic phase was washed with aq sat. $\mathrm{NaCl}(10 \mathrm{~mL})$ and dried over $\mathrm{Na}_{2} \mathrm{SO}_{4}$. Removal of solvent under reduced pressure and subsequent column chromatography on silica gel afforded dodecan-2-one $\mathbf{3 a}$ (76 $\mathrm{mg}, 82 \%$ ) as colorless liquid.

Dodecan-2-one (3a): ${ }^{3}$ Colorless liquid; ${ }^{1} \mathrm{H}$ NMR $\left(200 \mathrm{MHz}, \mathrm{CDCl}_{3}\right) \delta 0.88(\mathrm{t}, J=6.5 \mathrm{~Hz}, 3 \mathrm{H})$, $1.20-1.38(\mathrm{~m}, 14 \mathrm{H}), 1.50-1.66(\mathrm{~m}, 2 \mathrm{H}), 2.14(\mathrm{~s}, 3 \mathrm{H}), 2.42(\mathrm{t}, J=7.4 \mathrm{~Hz}, 2 \mathrm{H}) ;{ }^{13} \mathrm{C}$ NMR $(50 \mathrm{MHz}$, $\left.\mathrm{CDCl}_{3}\right) \delta 14.1,22.7,23.9,29.2,29.3,29.40,29.48,29.55,29.8,31.9,43.8,209.2$; IR (neat) 2925, 2854, $1718,1466,1411,1359,1162 \mathrm{~cm}^{-1}$.

Decan-2-one (3b): ${ }^{4}$ Colorless liquid; ${ }^{1} \mathrm{H}$ NMR $\left(200 \mathrm{MHz}, \mathrm{CDCl}_{3}\right) \delta 0.88(\mathrm{t}, J=6.6 \mathrm{~Hz}, 3 \mathrm{H})$, 1.20-1.36 (m, 10H), 1.50-1.64 (m, 2H), $2.14(\mathrm{~s}, 3 \mathrm{H}), 2.42(\mathrm{t}, J=7.4 \mathrm{~Hz}, 2 \mathrm{H}) ;{ }^{13} \mathrm{C} \mathrm{NMR}(50 \mathrm{MHz}$, $\left.\mathrm{CDCl}_{3}\right) \delta 14.1,22.6,23.9,29.1,29.2,29.4,29.8,31.8,43.8,209.2$; IR (neat) 2928, 2855, 1715, 1465, $1412,1360,1163 \mathrm{~cm}^{-1}$.

Undecan-2-one (3c): ${ }^{5}$ Colorless liquid; ${ }^{1} \mathrm{H}$ NMR $\left(200 \mathrm{MHz}, \mathrm{CDCl}_{3}\right) \delta 0.88(\mathrm{t}, J=6.4 \mathrm{~Hz}, 3 \mathrm{H})$, $1.20-1.38(\mathrm{~m}, 12 \mathrm{H}), 1.50-1.64(\mathrm{~m}, 2 \mathrm{H}), 2.14(\mathrm{~s}, 3 \mathrm{H}), 2.42(\mathrm{t}, J=7.3 \mathrm{~Hz}, 2 \mathrm{H}) ;{ }^{13} \mathrm{C}$ NMR $(50 \mathrm{MHz}$, $\left.\mathrm{CDCl}_{3}\right) \delta 14.0,22.6,23.8,29.1,29.2,29.4,29.7,31.8,43.7,209.1$; IR (neat) 2926, 2854, 1716, 1466, $1411,1359,1162 \mathrm{~cm}^{-1}$.

Tetradodecan-2-one (3d): White solids; ${ }^{1} \mathrm{H}$ NMR $\left(200 \mathrm{MHz}, \mathrm{CDCl}_{3}\right) \delta 0.88$ (t, $\left.J=6.5 \mathrm{~Hz}, 3 \mathrm{H}\right)$, $1.20-1.36(\mathrm{~m}, 18 \mathrm{H}), 1.50-1.64(\mathrm{~m}, 2 \mathrm{H}), 2.13(\mathrm{~s}, 3 \mathrm{H}), 2.42(\mathrm{t}, J=7.4 \mathrm{~Hz}, 2 \mathrm{H}) ;{ }^{13} \mathrm{C}$ NMR $(50 \mathrm{MHz}$, $\left.\mathrm{CDCl}_{3}\right) \delta 14.1,22.7,23.9,29.2,29.3,29.4,29.5,29.6,29.8,31.9,43.8,209.1$; IR (KBr) 2917, 2849, 1712 , $1464,1414,1376,1168 \mathrm{~cm}^{-1}$.

Methyl 10-oxoundecanoate (3e): ${ }^{6}$ Colorless liquid; ${ }^{1} \mathrm{H}$ NMR $\left(200 \mathrm{MHz}, \mathrm{CDCl}_{3}\right) \delta 1.24-1.34(\mathrm{~m}, 8 \mathrm{H})$, $1.50-1.66(\mathrm{~m}, 4 \mathrm{H}), 2.13(\mathrm{~s}, 3 \mathrm{H}), 2.30(\mathrm{t}, J=7.5 \mathrm{~Hz}, 2 \mathrm{H}), 2.42(\mathrm{t}, J=7.4 \mathrm{~Hz}, 2 \mathrm{H}), 3.67(\mathrm{~s}, 3 \mathrm{H}) ;{ }^{13} \mathrm{C} \mathrm{NMR}$ $\left(50 \mathrm{MHz}, \mathrm{CDCl}_{3}\right) \delta 23.8,24.9,29.0,29.1,29.8,34.0,43.7,51.4,174.1,209.1$; IR (neat) 2931, 2856, $1715,1437,1361,1171 \mathrm{~cm}^{-1}$.

4-Phenylbutan-2-one (3f): ${ }^{5}$ Colorless liquid; ${ }^{1} \mathrm{H}$ NMR $\left(200 \mathrm{MHz}, \mathrm{CDCl}_{3}\right) \delta 2.15$ (s, 3H), 2.72-2.94 (m, 4H), 7.16-7.32 (m, 5H); ${ }^{13} \mathrm{C}$ NMR (50 MHz, $\left.\mathrm{CDCl}_{3}\right) \delta 29.7,30.0,45.1,126.0,128.1,128.3,140.8$,

(3) Tokuyasu, T.; Kunikawa, S.; Masuyama, A.; Nojima, M. Org. Lett. 2002, 4, 3595-3598.

(4) Ragagnin, G.; Betzemeier, B.; Quici, S.; Knochel, P. Tetrahedron 2002, 58, 3985-3991.

(5) Gerard, J.; Hevesi, L. Tetrahedron 2004, 60, 367-381.

(6) Schultz, S.; Toft, S., Tetrahedron 1993, 49, 6805-6820. 
207.7; IR (KBr) 3062, 3028, 2925, 1717, 1603, 1496, 1453, 1409, 1358, $1162 \mathrm{~cm}^{-1}$.

10-Oxoundecanal (3g): ${ }^{7}$ Colorless liquid; ${ }^{1} \mathrm{H}$ NMR $\left(200 \mathrm{MHz}, \mathrm{CDCl}_{3}\right) \delta 1.24-1.36(\mathrm{~m}, 8 \mathrm{H})$, $1.50-1.68(\mathrm{~m}, 4 \mathrm{H}), 2.13(\mathrm{~s}, 3 \mathrm{H}), 2.42(\mathrm{t}, J=7.4 \mathrm{~Hz}, 4 \mathrm{H}), 9.76(\mathrm{~s}, 1 \mathrm{H}) ;{ }^{13} \mathrm{C} \mathrm{NMR}\left(50 \mathrm{MHz}, \mathrm{CDCl}_{3}\right) \delta 22.0$, 23.7, 29.0, 29.1, 29.8, 43.7, 43.8, 202.7, 209.1; IR (KBr) 2930, 2856, 2720, 1717, 1464, 1411, 1361, 1166 $\mathrm{cm}^{-1}$.

4-Benzyloxybutan-2-one (3h): ${ }^{8}$ Colorless liquid; ${ }^{1} \mathrm{H}$ NMR (200 MHz, $\left.\mathrm{CDCl}_{3}\right) \delta 2.18$ (s, 3H), $2.72(\mathrm{t}$, $J=6.3 \mathrm{~Hz}, 2 \mathrm{H}), 3.74(\mathrm{t}, J=6.3 \mathrm{~Hz}, 2 \mathrm{H}), 4.51(\mathrm{~s}, 2 \mathrm{H}), 7.29-7.37(\mathrm{~m}, 5 \mathrm{H}) ;{ }^{13} \mathrm{C} \mathrm{NMR}\left(50 \mathrm{MHz}, \mathrm{CDCl}_{3}\right) \delta$ 30.4, 43.7, 65.1, 73.1, 127.5, 128.2, 137.9, 207.0; IR (neat) 3063, 3031, 2867, 1715, 1496, 1421, 1364 , $1233,1171,1105 \mathrm{~cm}^{-1}$.

\section{Electrooxidation of Palladium Acetate in the Presence of TEMPO}

The anodic oxidation was carried out in an H-type divided cell (glass filter) equipped with two platinum electrodes $\left(1.5 \times 2.0 \mathrm{~cm}^{2}\right)$. In the anodic chamber was placed a solution of $\operatorname{Pd}(\mathrm{OAc})_{2}(225 \mathrm{mg}$, $1.0 \mathrm{mmol})$ and TEMPO (469 mg, $3.0 \mathrm{mmol})$ in $0.1 \mathrm{M} \mathrm{Bu}_{4} \mathrm{NBF}_{4} / \mathrm{CH}_{3} \mathrm{CN}(15 \mathrm{~mL})$. In the cathodic chamber was placed $0.1 \mathrm{M} \mathrm{Bu}_{4} \mathrm{NBF}_{4} / \mathrm{CH}_{3} \mathrm{CN}(15 \mathrm{~mL})$. Under argon, the constant current electrolysis (10 $\mathrm{mA}$ ) was carried out at room temperature with magnetic stirring until $2.4 \mathrm{~F} / \mathrm{mol}$ of electricity was passed. After the electrolysis, to the combined solution of the anodic and cathodic chambers was added $\operatorname{dry} \mathrm{Et}_{2} \mathrm{O}$ $(30 \mathrm{~mL})$. The resulting pale yellow precipitate was washed with dry $\mathrm{Et}_{2} \mathrm{O}$ and dried under reduced pressure to afford $\left[\mathrm{Pd}\left(\mathrm{CH}_{3} \mathrm{CN}\right)_{4}\right.$ (tempo)][BF $]$ (237 mg, 46\%). IR (KBr) 3443, 2962, 2188, 1636, 1417 $\mathrm{cm}^{-1}$. Anal. Calcd for $\mathrm{C}_{17} \mathrm{H}_{30} \mathrm{BF}_{4} \mathrm{~N}_{5} \mathrm{OPd}$ : C, 39.75; H, 5.89; N, 13.63. Found: C, 39.32; H, 5.54; N, 13.66.

(7) McMurry, J. E.; Silvestri, M. G.; Fleming, M. P.; Hoz, T.; Grayston, M. W. J. Org. Chem. 1978, 43, 3249-3255. (8)Wabnitz, T. C.; Spencer, J. B. Org. Lett. 2003, 5, 2141-2144. 